\title{
On the impossibility of representing infinite utility streams
}

\author{
Juan Alfonso Crespo • Carmelo Nuñez • \\ Juan Pablo Rincón-Zapatero
}

\begin{abstract}
We introduce a new Pareto-type criterion for social welfare functions over infinite utility streams that is not necessarily sensitive to increments in just a finite number of components. We show that there is no social welfare function that satisfies both this criterion and Diamond's equity condition simultaneously. With our result, we extend the impossibility theorem of Basu and Mitra. Moreover, we show that, even under a weaker version of equity related to Zame's intergenerational equity condition, the impossibility results are obtained as well.
\end{abstract}

Keywords Social welfare function - Intergenerational equity · Pareto axiom . Overtaking criterion

\section{JEL Classification D63 $\cdot \mathrm{D} 71 \cdot \mathrm{D} 90$}

\section{Introduction}

Infinite utility streams are a useful tool to understand economic problems with an infinite time horizon. Introduced by Ramsey (1928), they have been used by Koopmans

\footnotetext{
The authors want to thank the anonymous referees for helpful comments. Juan Alfonso Crespo and Carmelo Nuñez are partially supported by MEC grant SEJ2007-67135. Juan Pablo Rincón-Zapatero is partially supported by the FEDER-MEC grant MTM2005-06534.

J. A. Crespo $(\varangle) \cdot$ C. Nuñez · J. P. Rincón-Zapatero

Departamento de Economía, Universidad Carlos III de Madrid, 28903 Getafe, Spain

e-mail: jacrespo@eco.uc3m.es

C. Nuñez

e-mail: cnunez@eco.uc3m.es

J. P. Rincón-Zapatero

e-mail: jrincon@eco.uc3m.es
} 
(1960); von Weizsäcker (1965) and Gale (1967) among others to study models of optimal growth, savings, taxation, and investment. For instance, Becker and Boyd (1997) and Dana et al. (2006) are good references on recent developments in these topics.

In the study of infinite utility streams, a challenging problem is the existence of a utility function representing the order given on the set of these streams. Such a function is called a social welfare function (SWF). A SWF is a rule that aggregates the consumption of all generations into a real number, preserving preferences imposed on the set $X$ of all infinite utility streams. In other words, a SWF assigns a level of welfare enjoyed by the whole society to an arbitrary sequence of consumption.

In Koopmans (1960); Diamond (1965) and Basu and Mitra (2003) it is shown that a SWF preserving the Pareto order is incompatible with an egalitarian treatment of all generations, in the sense that if one permutes the consumption levels of two generations, the level of welfare remains the same. More precisely, Koopmans shows that some form of impatience arises when the utility function satisfies recursive properties. Diamond proves that a Paretian and egalitarian continuous utility function never exists under certain restrictions on the metric defined for the set $X$. Finally, Basu and Mitra prove that, independently of the topology chosen for $X$, it is impossible to construct an utility function that satisfies the strong Pareto $^{1}$ axiom and that is also egalitarian.

Our starting point is the following observation: the strong Pareto axiom demands that, for a given consumption stream, an increment in consumption of just one generation increases the value of this new stream over the original. This seems to be too strict economically because, intuitively, the welfare of a society with infinitely many generations should not be influenced by the utility of just one generation.

Basu and Mitra introduce weaker versions of the strong Pareto axiom. They call them the dominance axiom and the partial Pareto axiom in Basu and Mitra (2003, 2008), respectively. In both cases they prove that when the consumption of each generation can be chosen in the interval $[0,1]$, then an impossibility result arises if one imposes Diamond's equity. Both axioms impose that increments in either finitely many or in all components imply more utility. Nevertheless, nothing is imposed on intermediate cases, that is, where infinitely many components are increased and the others remain the same.

It seems to us that in an economy with infinitely many generations, Ramsey's idea of not assigning more importance to one generation than to others is preserved if the welfare of any one generation is negligible in comparison with the welfare of the whole economy. In consequence, we propose a criterion that we call the infinite Pareto principle, where only infinitely many increments affect the value of the SWF. More precisely, one consumption stream is valued more highly than another if infinitely many components of the first are of higher value than the respective components of the second and if the other generations remain the same.

Another point of view that can help to explain our criterion is the following. In terms of intergenerational justice, it seems to us that an increment in the accumulated consumption of all generations benefits the welfare of the whole society only if

\footnotetext{
${ }^{1}$ This seems to be the standard name for the axiom, while Basu and Mitra refer to it simply as Pareto.
} 
infinitely many generations enjoy this increment. In other words, if one can always find generations in the future (infinitely many, but not necessarily all) with a positive increment in their consumption. ${ }^{2}$

A second point to ponder is the following perspective on the idea of equity. We recall that Diamond's equity means that finite permutations in the utility streams give the same aggregated utility. Our idea is that this condition may be too strong because, in many frameworks, we are only interested in preserving the strict preferences by means of finite permutations. Consequently, we consider a weak equity condition on the $\mathrm{SWF}^{3}$ that avoids the constraint that equity imposes in cases of indifference and, at the same time, preserves the spirit of Ramsey and Gale, among others.

We show that, under our weaker hypotheses, Basu and Mitra's (2003) result remains true. We remark that, as in Basu and Mitra (2003), our result is independent of the topology assigned to the space of all utility streams.

\section{Infinite utility streams}

An infinite utility stream is an infinite sequence $x=\left(x_{1}, \ldots, x_{n}, \ldots\right)$ where $x_{i} \in A$, a subset of $\mathbb{R}$. In other words, $x \in A^{\mathbb{N}}$, the infinite Cartesian product of $A$. We will denote $X=A^{\mathbb{N}}$. We will assume from now on that $A$ contains at least two points; if not, the problem is tautological.

A social welfare function (SWF) is a function $W: X \rightarrow \mathbb{R}$ such that, for a given element $x \in X, W(x)$ measures the level of welfare that the sequence of consumptions $\left(x_{i}\right)$ produces in the whole society.

Several principles have been imposed on the SWFs: for instance, the strong Pareto and weak Pareto stated below. Moreover, we introduce a new intermediate principle that seems to us to be very natural.

Definition 2.1 (Strong Pareto principle) We say that $W$, a SWF, is strong Paretian if, given $x$ and $y$ in $X$, satisfying:

1. $x_{i} \geq y_{i}$ for all $i \in \mathbb{N}$, and

2. there exists $j \in \mathbb{N}$ such that $x_{j}>y_{j}$,

then $W(x)>W(y)$.

If $x$ and $y$ satisfy conditions (1) and (2) in the above definition we will write $x \succ_{\mathcal{P}} y$.

Definition 2.2 (Weak Pareto principle) We say that $W$, a SWF, is weak Paretian if given $x, y \in X$ such that $x_{i}>y_{i} \forall i \in \mathbb{N}$, then $W(x)>W(y)$.

When $x$ and $y$ satisfy this condition we will use the notation $x \succ_{\mathcal{W}} y$.

A strong Paretian SWF is sensitive to increments in at least one component. As discussed in the introduction, one unit of consumption should not, in principle, affect

2 Note the difference between our criterion and the so-called Hammond Equity for the Future, cf. Banerjee (2006): If $x, y \in X$, such that $x_{1}>y_{1}>u>v, x=\left(x_{1}, v, v, \ldots\right)$ and $y=\left(y_{1}, u, u, \ldots\right)$ then, $W(y) \geq W(x)$.

3 Zame (2007) uses strict preferences that display intergenerational equity. 
the global welfare of an economy, where infinitely many consumers are assumed. ${ }^{4}$ On the contrary, a weak Paretian SWF is only sensitive to increments in all the components. For this reason we introduce the following intermediate Pareto criterion.

Definition 2.3 (Infinite Pareto principle) We say that $W$, a SWF, is infinite Paretian if given $x, y \in X$ such that:

1. $x_{i} \geq y_{i} \forall i \in \mathbb{N}$, and

2. there exists $M \subset \mathbb{N}$ with $\sharp M=\infty$ such that $x_{j}>y_{j} \forall j \in M$,

then $W(x)>W(y)$.

If $x$ and $y$ satisfy conditions (1) and (2) in the above definition we will write $x \succ_{\mathcal{I}} y$.

An alternative way to define this new principle is to say that for $x$ and $y$ satisfying:

1. $x_{j} \geq y_{j}, \forall j$ and

2. there exists $\left(s_{n}\right)_{n \geq 1}$, a strictly increasing sequence of natural numbers, such that $x_{s_{j}}>y_{s_{j}}$

then $W(x)>W(y)$.

An infinite Paretian SWF is sensitive to increments in infinitely many components but, a priori, increments in just a finite number of them do not imply a variation in the value of the SWF.

Obviously, if $W$ is strong Paretian, then $W$ is infinite Paretian and, analogously, if $W$ is infinite Paretian, then $W$ is weak Paretian, but the converses are not true as the following SWFs show.

Example 2.4

(a) Consider $A \subset \mathbb{R}$ and let

$$
W(x)=\sum_{n>1} \frac{1}{2^{n}} \arctan \left(x_{n}\right) .
$$

This is obviously an infinite Paretian SWF but it does not satisfy the strong Pareto principle.

(b) Suppose $A \subset \mathbb{R}$ any lower-bounded, closed and discrete set and let

$$
W(x)=\inf _{n \geq 1} x_{n}
$$

the Rawlsian SWF. This is obviously a weak Paretian SWF but it does not satisfy the infinite Pareto principle.

Now we recall the concept of equity, as introduced by Diamond (1965), to ensure intergenerational justice. Observe the contrast with Pareto principles, which deal with efficiency.

4 This point of view agrees with Aumann's (1964), where an individual in an economy with a continuum of agents is negligible. This fact has also been pointed out by Lauwers (1998). 
Definition 2.5 Let $W$ be a SWF. We say that $W$ is egalitarian (or that $W$ satisfies the equity condition) if, for all $x, y$ in $X$ such that:

1. $x$ and $y$ differ only in periods $i, j$, and

2. $x_{i}=y_{j}, x_{j}=y_{i}$,

then $W(x)=W(y)$.

This is equivalent to saying that the value of $W(x)$ is not affected by finite permutations of the components of $x$. For instance, the SWF in Example 2.4(b) is egalitarian, but the one in Example 2.4(a) is not.

Remark 2.6 Assume that $W$ is infinite Paretian and egalitarian. If $x \succ_{\mathcal{I}} y$, then $W(\sigma x)>W(\tau y)$, where $\sigma x$ and $\tau y$ are obtained applying the finite permutations $\sigma$ and $\tau$ to the components of $x$ and $y$, respectively.

\section{The impossibility theorem}

As is shown in Example 2.4(b), there are weak Paretian and egalitarian SWFs under some mild conditions on the consumption domain. Basu and Mitra (2003) prove that there does not exist any strong Paretian and egalitarian SWF without restrictions on the domain. In this section we will prove that it is also impossible to construct an infinite Paretian and egalitarian SWF for any domain.

Let us recall the immersion of the interval $(0,1)$ in $\{0,1\}^{\mathbb{N}}$, given by Sierpiński (1965), which was used in Basu and Mitra (2003) to prove their impossibility theorem. Set $q_{1}, \ldots, q_{n}, \ldots$ an enumeration of $\mathbb{Q} \cap(0,1)$. For $r \in(0,1)$, let the (infinite) sequence $i(r) \in\{0,1\}^{\mathbb{N}}$ be defined as follows

$$
i(r)_{n}= \begin{cases}1 & \text { if } q_{n}<r \\ 0 & \text { if } q_{n} \geq r\end{cases}
$$

\section{Remark 3.1}

1. $i(r)$ contains infinitely many ones and infinitely many zeros. This is because there are infinitely many rationals $q_{n}$ in the interval $(0, r)$ (so $\left.i(r)_{n}=1\right)$ as well as infinitely many $q_{m}$ in $[r, 1)$ (and hence $i(r)_{m}=0$ ).

2. If $r<s$ are two numbers in $(0,1)$ then $i(r) \prec_{\mathcal{I}} i(s)$ in $\{0,1\}^{\mathbb{N}}$. Indeed, if $i(r)_{n}=1$ one has $q_{n}<r<s$ and hence $i(s)_{n}=1$. On the other hand, the interval $[r, s)$ contains infinitely many rationals $q_{m}$, so in the components associated to those rationals $i(r)_{m}=0$ while $i(s)_{m}=1$.

Proposition 3.2 Let $W$ be an infinite Paretian SWF on $X$. Then, the composite $f=$ $W \circ i:(0,1) \rightarrow(0,1)$ has, at most, a countable set of discontinuities.

Proof The composite $f=W \circ i$ is a strictly increasing function from $(0,1)$ to $(0,1)$ because, if $r<s$, then $i(r) \prec_{\mathcal{I}} i(s)$ by Remark 3.1 (2) and, if $x \prec_{\mathcal{I}} y$, then $W(x)<W(y)$ since $W$ is infinite Paretian. Hence, $f$ could have at most a countable set of discontinuities. 
Our main result extends the impossibility theorem of Basu and Mitra (2003) to our framework. In fact, our proof is inspired by theirs, in the sense that we use Sierpinski's construction to produce a strictly increasing function with a non-countable number of discontinuities.

\section{Theorem 3.3 There does not exist an egalitarian and infinite Paretian SWF.}

Proof First of all, observe that it is enough to prove the result for $X=\{0,1\}^{\mathbb{N}}$. Indeed, if $X$ has at least two elements and $W$ is a SWF that satisfies the hypotheses of the theorem, then $W$ has to satisfy them when we restrict to sequences with components taken at these two points.

We will show that, if such a $W$ exists, then the composite $f=W \circ i$ is discontinuous at every $r \in(0,1)$, contradicting Proposition 3.2.

Fix $r \in(0,1)$ and consider $i(r)$. We are going to construct an element of $X, i(r)^{+}$, such that $W(i(r))<W\left(i(r)^{+}\right)<W(i(s))$ for all $s \in(r, 1)$.

Let $q_{1}, \ldots, q_{k}, \ldots$ be the enumeration of the rationals in $(0,1)$ used to define the immersion $i$. Consider a strictly decreasing sequence of rational numbers $\left(z_{j}\right)_{j \geq 1}$ in $(0,1)$, converging to $r$, and define $i(r)^{+}$as follows:

$$
i(r)_{n}^{+}=\left\{\begin{array}{ll}
1 & \text { if } q_{n}<r \\
1 & \text { if } q_{n}=z_{j} \\
0 & \text { otherwise }
\end{array} \text { for some } j\right.
$$

So, if $q_{n}<r$, we have $i(r)_{n}^{+}=1=i(r)_{n}$. If $q_{n}=z_{j}$ for some $j$, then $i(r)_{n}=0<$ $1=i(r)_{n}^{+}$. Finally, if $q_{n} \geq r, q_{n} \neq z_{j}$ for all $j$, then $i(r)_{n}=0=i(r)_{n}^{+}$. In other words, $i(r) \prec_{\mathcal{I}} i(r)^{+}$, because $\left(z_{j}\right)_{j \geq 1}$ is an infinite sequence of rational numbers bigger than $r$.

On the other hand, let $r<s<1$ and consider $i(s)$. Then $W\left(i(r)^{+}\right)<W(i(s))$. Indeed, as $\left(z_{j}\right)_{j \geq 1}$ converges to $r$, there exists $n_{0}$ such that $z_{n}<s$ for all $n>$ $n_{0}$, hence there is only a finite number of components $n$ such that $i(r)_{n}^{+}=1$ and $i(s)_{n}=0$, precisely those $n$ such that $q_{n}$ is equal to some $z_{j}$ for $j=1, \ldots, n_{0}$. Denote these components by $i(s)_{a_{1}}, \ldots, i(s)_{a_{n_{0}}}$. Moreover, there are infinitely many rational numbers in $(r, s)$ that are not in $\left(z_{j}\right)_{j \geq 1}$, hence there are infinitely many components $n$ such that $i(s)_{n}=1$ and $i(r)_{n}^{+}=0$. Denote these components by $i(s)_{b_{j}}$ with $j \in \mathbb{N}$. Now permute the components $i(s)_{a_{j}}$ with the components $i(s)_{b_{j}}$ for $j=1, \ldots, n_{0}$ and call this new vector $\sigma i(s)$. By construction $i(r)^{+} \prec_{\mathcal{I}} \sigma i(s)$ and, using first the infinite Pareto principle and second the Equity condition, one obtains $W\left(i(r)^{+}\right)<W(\sigma i(s))=W(i(s))$.

Observe that the construction of $i(r)^{+}$does not depend on $s$. So, for any given $s \in(0,1)$, with $r<s$, one has $W(i(r))<M<W(i(s))$, where $M=W\left(i(r)^{+}\right)$. Then, $W \circ i$ is discontinuous at every $r \in(0,1)$.

Corollary 3.4 (Basu and Mitra 2003). There does not exist an egalitarian and strong Paretian SWF.

We can extend the previous results to other known criteria, such as von Weiszäcker's overtaking criterion. Following Asheim and Tungodden (2004), we recall only the 
strict preference of this criterion. We say that $W$ preserves the overtaking criterion if $x \prec o y$ implies $W(x)<W(y)$, where

$$
x \prec o \quad y \Leftrightarrow \exists n_{0} \quad \text { such that } \sum_{k=1}^{n} x_{k}<\sum_{k=1}^{n} y_{k}, \quad \forall n>n_{0} .
$$

Observe that $x \prec_{\mathcal{P}} y$ implies $x \prec_{O} \quad y$. Hence, if an egalitarian SWF preserves the overtaking criterion, then it has to be strong Paretian, which is impossible by Corollary 3.4. We have thus obtained the following result.

Corollary 3.5 There does not exist an egalitarian SWF preserving von Weiszäcker's overtaking criterion.

\section{Weakening equity}

Our main result in this paper (as well as in Theorems 1 and 2 of Basu-Mitra's paper) is that Diamond's equity is too strong a condition to be compatible with both the infinite Pareto principle and the strong Pareto principle. One might think that these impossibility results arise because equity among generations deals with indifference relations, while the Pareto principles deal with strict preferences. Zame (2007) works with a notion of intergenerational equity applied to the case of strict preferences. Precisely, a strict preference relation $\succ$ displays intergenerational equity if, given $x \succ y$, then $\sigma x \succ \tau y$ for any finite permutations $\sigma$ and $\tau$ of the components of $x$ and $y$. In a parallel way, we define a weaker version of intergenerational equity than the one defined in Sect. 2.

Definition 4.1 Let $W: X \rightarrow \mathbb{R}$ be a SWF and $\succ$ an irreflexive preference on $X$. We say that $W$ is weak egalitarian (with respect to $\succ$ ) if, for every $x$ and $y$ in $X$ with $x \succ y$, then $W(\sigma x)>W(\tau y)$, for any $\sigma$ and $\tau$ finite permutations of the components of $x$ and $y$.

Observe that an egalitarian strong (respectively infinite) Paretian SWF satisfies this condition for the irreflexive preference $\prec_{\mathcal{P}}$ (respectively $\prec_{\mathcal{I}}$ ) but, obviously, the converse is not true. In case that we have $W$, a SWF which is strong Paretian and weak egalitarian with respect to $\prec \mathcal{P}$, we will just say that $W$ is a weak egalitarian strong Paretian SWF. In the same way, we will talk about a weak egalitarian infinite Paretian SWF.

The goal of this section is to prove that, even under this weak version of equity, the impossibility results appear for both strong and infinite Paretian SWFs.

First of all, we will show a set of sequences that are comparable by means of any SWF that is either weak egalitarian strong Paretian or weak egalitarian infinite Paretian. This will be the case if we impose Diamond's equity on $W$ and this SWF is either strong Paretian or infinite Paretian.

Lemma 4.2 Let $W$ be a SWF over X. Assume that $W$ is either a weak egalitarian strong Paretian or a weak egalitarian infinite Paretian SWF. Let $a, b \in A$ and $x, y \in\{a, b\}^{\mathbb{N}}$ satisfying: 
1. $y_{i}<x_{i}$ for, at most, finitely many $i$, and

2. $x_{j}<y_{j}$ for infinitely many $j$ in $\mathbb{N}$;

then $W(x)<W(y)$.

Proof Assume $a<b$. Under the assumptions of the Lemma, there exists $\left(s_{n}\right)_{n \geq 1}$, a strictly increasing sequence in $\mathbb{N}$ such that $x_{s_{j}}=a<b=y_{s_{j}}$ and, only for certain $r_{1}, r_{2}, \ldots, r_{k}$ in $\mathbb{N}$, one has $y_{r_{j}}=a<b=x_{r_{j}}$. For $j=1, \ldots, k$, rearranging the components $x_{r_{j}}$ into the components $x_{s_{j}}$ and vice versa is just a finite permutation of the components of $x$. Call this new vector $\sigma x=\left((\sigma x)_{1}, \ldots,(\sigma x)_{n}, \ldots\right)$. By construction $y_{i}-(\sigma x)_{i} \geq 0$ for all $i$ and $y_{s_{j}}-(\sigma x)_{s_{j}}=b-a$ for $j=k+1, \ldots$, hence $\sigma x \prec_{\mathcal{I}} y$ (and also $\sigma x \prec_{\mathcal{P}} y$ ). Then, using the weak equity condition, we obtain $W(x)<W(y)$, because $\sigma x$ is a finite permutation of $x$.

If two sequences $x, y$ in $\{a, b\}^{\mathbb{N}}$ satisfy conditions (1) and (2) in Lemma 4.2 we will write $x \prec \mathcal{Q} y$.

The converse of this result is also true when we restrict the domain to a set with only two points.

Lemma 4.3 Let $a, b$ be two real numbers and $X=\{a, b\}^{\mathbb{N}}$. Let $W$ be a SWF on $X$ such that, if $x$ and $y$ satisfy $x \prec \mathcal{Q} y$, one has $W(x)<W(y)$. Then, $W$ is a weak egalitarian infinite Paretian SWF.

Proof Under the conditions of the Lemma, $W$ is obviously infinite Paretian. Now consider $x \prec \mathcal{I} y$. It is also evident that $\sigma x \prec \mathcal{Q} \tau y$ for any pair of finite permutations $\sigma$ and $\tau$. Hence $W(\sigma x)<W(\tau y)$.

Putting together the above results, one has the following characterization of weak egalitarian infinite Paretian SWFs defined over binary sets, by means of the irreflexive preference relation $\prec \mathcal{Q}$.

Proposition 4.4 Let $a, b$ be two real numbers and $X=\{a, b\}^{\mathbb{N}}$. Let $W$ be a SWF on $X$. Then, $W$ is a weak egalitarian infinite Paretian $S W F$ if and only if, for any $x$ and $y$ with $x \prec \mathcal{Q} y$, one has $W(x)<W(y)$.

Finally, we show that, even under the weakest egalitarian hypotheses introduced in this section, the impossibility result also holds.

Theorem 4.5 There does not exist a weak egalitarian and infinite Paretian SWF.

Proof Suppose that $W$ satisfies the hypotheses of the Theorem. Call $\widetilde{W}$ the restriction of $W$ to the set $\{a, b\}^{\mathbb{N}}$, where $a<b$ are two arbitrary points in $A$. Then, $\widetilde{W}$ also has to satisfy the conditions of the Theorem. Now Proposition 4.4 implies that, for any $x$ and $y$ in $\{a, b\}^{\mathbb{N}}$, if $x \prec \mathcal{Q} y$ one has $\widetilde{W}(x)<\widetilde{W}(y)$. We will see that this is impossible. Assuming without loss of generality that $a=0$ and $b=1$, then $X=\{0,1\}^{\mathbb{N}}$.

The proof of Theorem 3.3 follows as well in this case, in fact, in an easier way. Recall that, using Sierpinski's immersion, we assigned to every $r \in(0,1)$ a sequence $i(r) \in X$. We then constructed a sequence $i(r)^{+}$satisfying $i(r) \prec \mathcal{I} i(r)^{+}$and hence $i(r) \prec_{\mathcal{Q}} i(r)^{+}$. Now, for any $s$ with $r<s<1$, we proved that $i(r)_{m}^{+}>i(s)_{m}$ 
for just finitely many values of $m$. Moreover, $i(r)_{j}^{+}<i(s)_{j}$ for infinitely many $j$. Then, $i(r)^{+} \prec \mathcal{Q} i(s)$ and, hence, $\widetilde{W}(i(r))<\widetilde{W}\left(i(r)^{+}\right)<\widetilde{W}(i(s))$, arriving at a contradiction.

As any weak egalitarian strong Paretian SWF is automatically weak egalitarian infinite Paretian, the following result follows.

Corollary 4.6 There does not exist a weak egalitarian and strong Paretian SWF.

\section{Concluding Remarks}

It is proved in Basu and Mitra (2003) that there does not exist any social welfare function which satisfies the strong Pareto and intergenerational equity axioms. When they relax the strong Pareto axiom and arrive at the weaker version they call the dominance axiom, they recognize on page 1561 that "It is not as if we wish to recommend the use of such a weak form of the Pareto condition...". So, they left open the following problem: is it possible to have another weaker and economically acceptable version of the Pareto axiom from which the impossibility result follows? Inspired by Aumann's model of competitive markets with a continuum of agents, we propose a modification of the Pareto axiom aimed at deriving the negligible utility of a single generation. In the same way, Lauwers (1998) criticizes the relevance of a single generation, which is the key point of the strong Pareto axiom.

On the other hand, Zame (2007) introduces a notion of equity for strict preferences. We follow this concept in order, first, to weaken Basu-Mitra's hypotheses and, second, to give an idea of equity that is compatible with the impossibility of a single generation having veto power.

In consequence, our aim is to reflect in the axioms an ordering where the welfare of the whole society is not necessarily modified by a change in a single generation (and by extension, of any finite number of generations), but increments in infinitely many generations imply an increasing level of welfare if no generation experiences a loss.

Our line of attack is twofold: (a) to introduce a weaker version of the strong Pareto principle (that we call the infinite Pareto principle), and (b) to adapt Zame's weak equity condition. We have shown that, even under these hypotheses, Basu and Mitra (2003) impossibility result remains. We hope that our hypotheses will be mathematically simpler and economically more useful than Basu-Mitra's dominance or partial Pareto axioms.

\section{References}

Asheim, G., Tungodden, B.: Resolving distributional conflicts between generations. Econom Theory 24(1), 221-230 (2004)

Aumann, R.J.: Markets with a continuum of traders. Econometrica 32, 39-50 (1964)

Banerjee, K.: On the equity-efficiency trade off in aggregating infinite utility streams. Econ Lett 93, 63-67 (2006)

Basu, K., Mitra, T.: Aggregating infinite utility streams with intergenerational equity: the impossibility of being Paretian. Econometrica 71(5), 1557-1563 (2003) 
Basu, K., Mitra, T.: Possibility theorems for aggregating infinite utility streams, Intergenerational equity and sustainability. Roemer, J., Suzumura, K. (eds.) Palgrave. Available at http://www.arts.cornell.edu/ econ/cae/05-05.pdf (Forthcoming) (2008)

Becker, R.A., Boyd, J.H. III.: Capital Theory, Equilibrium Analysis and Recursive Utility. Malden: Basil Blackwell Publishers (1997)

Dana, R.A., Le Van, C., Mitra, T., Nishimura K. (eds.):. Handbook on Optimal Growth 1. Discrete time. Berlin, Springer (2006)

Diamond, P.A.: The evaluation of infinite utility streams. Econometrica 33, 170-177 (1965)

Koopmans, T.C.: Stationary ordinal utility and impatience. Econometrica 28, 287-309 (1960)

Lauwers, L.: Intertemporal objective functions: Strong Pareto versus anonymity. Math Soc Sci 35(1), 37-55 (1998)

Ramsey, F.P.: A mathematical theory of saving. Econ J 38(52), 543-559 (1928)

Sierpiński, W.: Cardinal and Ordinal Numbers, 2nd revised edition. Monografie Matematyczne, vol. 34. Państowe Wydawnictwo Naukowe, Warsaw (1965)

von Weizsächer, C.C.: Existence of optimal programs of accumulation for an infinite time horizon. Rev Econ Stud 32(2), 85-104 (1965)

Zame, W.R.: Can intergenerational equity be operationalized? Theor Econ 2, 187-202 (2007) 